\title{
THE CLINICALLY IMPORTANT CHANGES PHYSICAL FUNCTION WERE NOT ASSOCIATED WITH CLINICALLY IMPORTANT CHANGES IN MUSCLE STRENGTH OR PHYSICAL PERFORMANCE OVER TIME - A COHORT STUDY
}

Rafaela Cavalheiro do Espírito Santo ${ }^{1, *}$, Joshua F Baker², Jordana Miranda de Souza Silva³ ${ }^{3}$ Lidiane Isabel Filippin 4 , Juliana Katarina Schoer Portes ${ }^{1}$, Claiton Viegas Brenol ${ }^{1}$, Rafael Mendonça da Silva Chakr ${ }^{1}$, Ricardo Machado Xavier ${ }^{1}$

1.Hospital de Clínicas de Porto Alegre, Porto Alegre (RS), Brazil; 2.University of Pennsylvania, Philadelphia (PA), United States of America; 3.University Hospital Münster, Münster (North Rhine-Westphalia), Germany; 4.Universidade La Salle, Canoas (RS), Brazil.

*Corresponding author: rcsanto@hcpa.edu.br

\section{BACKGROUND}

The health assessment questionnaire disability index (HAQ) is negatively associated with muscle strength by handgrip test and positively associated with physical performance by timed up and go test (TUG test) in patients with rheumatoid arthritis (RA). Although the HAQ score may improve during treatment of inflammatory arthritis, the association between the self-reported HAQ scores and objective assessments of muscle strength or physical performance over time has not been clearly defined. Therefore, we aimed to evaluate the cross-sectional and longitudinal associations between self-reported physical function and clinical features, muscle strength and physical performance in two independent cohorts from North and South America.

\section{METHODS}

In a prospective cohort design, two independent RA cohorts were retrospectively analyzed. Physical function was assessed by HAQ. Disease activity was assessed by the disease activity score in 28 joint (DAS-28-CRP). Pain was assessed by the visual analog scale for pain (VAS pain). Muscle strength was assessed by handgrip test and chair test. Physical performance was assessed by TUG test and the short physical performance battery (SPPB). The T test for independent samples, Mann-Whitney $U$ test of independent samples, Pearson's chi-squared test, Spearman correlation coefficients was performed.

\section{RESULTS}

A total of 205 RA patients were studied (North American Cohort [ $n=115]$; Brazilian Cohort [ $n=90]$ ). Brazilian men had greater HAQ than North American men ( $p<0.001)$, while HAQ was not statistically different among women $(p=0.15)$. Brazilian women had lower muscle strength than North American women $(p<0.05)$, while Brazilian men showed less muscle strength than North American men $(p<0.05)$. Poor physical function as measured by HAQ was closely associated with disease activity, pain, muscle strength, and physical performance in both cohorts $(p<0.001)$. However, clinically important changes physical function were not associated with clinically important changes in muscle strength or physical performance over time $(p>0.05)$.

\section{CONCLUSION}

These observations suggest geographic differences in self-reported and directly assessed measures of physical performance, as well as a disconnect between changes in self-report of disability over time in patients with RA compared to more direct assessments of physical function.

\section{KEYWORDS}

Rheumatoid arthritis, Physical function, Muscle strength, Physical performance. 